\title{
Intestinal Stoma Obstruction, CTCAE
}

National Cancer Institute

\section{Source}

National Cancer Institute. Intestinal Stoma Obstruction, CT CAE. NCI Thesaurus. Code C143593.

A disorder characterized by blockage of the normal flow of the contents of the intestinal stoma. 